\title{
Ongoing Development of a Series Bosch Reactor System
}

\section{Authors: Morgan Abney, Matt Mansell, Sam DuMez, John Thomas, Charlie Cooper, David Long}

Future manned missions to deep space or planetary surfaces will undoubtedly require highly robust, efficient, and regenerable life support systems that require minimal consumables. To meet this requirement, NASA continues to explore a Bosch-based carbon dioxide reduction system to recover oxygen from $\mathrm{CO}_{2}$. In order to improve the equivalent system mass of Bosch systems, we seek to design and test a "Series Bosch" system in which two reactors in series are optimized for the two steps of the reaction, as well as to explore the use of in situ materials as carbon deposition catalysts. Here we report recent developments in this effort including assembly and initial testing of a Reverse Water-Gas Shift reactor (RWGSr) and initial testing of two gas separation membranes. The RWGSr was sized to reduce $\mathrm{CO}_{2}$ produced by a crew of four to carbon monoxide as the first stage in a Series Bosch system. The gas separation membranes, necessary to recycle unreacted hydrogen and $\mathrm{CO}_{2}$, were similarly sized. Additionally, we report results of preliminary experiments designed to determine the catalytic properties of Martian and Lunar regolith simulant for the carbon deposition step. 